\title{
An Adaptive Sliding Mode Differentiator for Actuator Oscillatory Failure Case Reconstruction
}

\author{
Halim Alwi, Christopher Edwards ${ }^{a}$ \\ ${ }^{a}$ Structures and Dynamics group, \\ College of Engineering, Mathematics and Physical Sciences, \\ University of Exeter, EX4 $4 Q F$, UK
}

\begin{abstract}
This paper proposes an adaptive sliding mode super-twisting differentiator which allows the gains to adapt based on the 'quality' of the sliding motion. A Lyapunov based analysis for the adaptive super-twisting scheme is presented to demonstrate its properties. As an example, the adaptive differentiator proposed in this paper has been used as part of a nonlinear FDI scheme for an Oscillatory Failure Case (OFC) in an actuator. The FDI scheme requires an estimate of the rod speed which is provided by the adaptive super-twisting differentiator. Due to the conditions in which the actuator operates, normally the differentiator gains are initialized at low values to ensure good rod speed estimation in fault free conditions. However for large amplitude/frequency OFCs, the gains must adapt in order to maintain sliding and provide a good estimation. Simulations on a high fidelity nonlinear aircraft benchmark model have been carried out for both liquid and solid OFCs.
\end{abstract}

Key words: fault detection and isolation, sliding modes, differentiators, oscillatory failure case.

\section{Introduction}

One of the areas of research which has benefited from advances in the area of higher order sliding modes, is the differentiation problem. In most engineering problems, finding the derivative of a signal is normally avoided - especially in the presence of noise. As highlighted in [14], in most cases, the problem of estimating the derivative of a signal is posed as an observer problem. The work of Levant described in [14] proposed a robust exact differentiator using a second order sliding mode technique. This has become known as 'super-twisting'. In particular the superiority of the super-twisting differentiator compared to conventional linear differentiators has been demonstrated. In the original work, geometrical arguments were used to prove the existence of sliding. However, recent theoretical developments described by Moreno and Osorio in [16] describe a Lyapunov approach. The use of Lyapunov based approaches has subsequently been investigated in [9], which proposes a variable gain formulation for the super-twisting algorithm, and [22] which proposes a super-twisting adaptive control formulation.

One application area in which observers (including slid-

Email addresses: H.Alwi@exeter.ac.uk (Halim Alwi), C.Edwards@exeter.ac.uk (Christopher Edwards). ing mode observers) are employed is the field of fault detection and isolation (FDI). The work of $[19,4]$ considers the use of higher order sliding mode differentiators for FDI. In [19], the differentiator generates a sufficient number of derivatives of the measurements to allow the unknown fault signals to be calculated. Derivatives of the measured outputs created by the exact differentiator from [14] are used in [6] to relieve the relative degree conditions associated with classic sliding mode observers [5]. Differentiators are also employed in [7] to provide finite time estimates of faults in linear systems. Higher order differentiators are also used for fault detection in nonlinear systems [8].

Early work on Oscillatory Failure Case (OFC) problems in [3] was motivated by the study of the effect of Electronic Flight Control Systems (EFCS) failures on the structural load on aircraft. The generation of erroneous sinusoidal signals from faulty electronic components (which is the main source of OFCs) propagate through the actuator control loop between the Flight Control Computer (FCC) and the control surface [11]. When coupled with the flexible modes of the structure, the oscillations can become unacceptable and cause high vibrations and loads due to resonance phenomenon. The OFC detection scheme currently in service on the AIRBUS A380 is discussed in [11]. The FDI scheme uses a filtered residual signal obtained from comparing the ac- 
tuator rod position and its analytical model-based estimate to confirm the presence of an OFC using oscillation counting. The work in [18] considers a linear observer for OFC detection and results are presented from a laboratory test rig for an inboard aileron actuation system. The work in $[1,2]$ proposes a nonlinear observer design to generate a residual together with an oscillation counting mechanism similar to the one in [11]. The nonlinear observer in [1] has two feedback terms which allows more degrees of freedom for the error system dynamics to be adjusted. Other recent results based on nonlinear observers are given in $[13,17]$.

This paper uses a novel adaptive super-twisting differentiator to create a nonlinear based method for reconstruction of the OFC signals. The idea is to obtain an expression for the OFC signal by manipulating the nonlinear mathematical model of the actuator. Except for the actuator rod speed, the parameters used in the manipulated nonlinear equations are available either through direct measurement or estimation. In this paper, the rod speed will be created using the adaptive sliding mode differentiator proposed in this paper. Sliding mode observers have previously been used to estimate the presence of oscillations in aerospace structures in [20]. The work in [20] uses a sliding mode observer to estimate the sloshing effect and exploits this estimate as part of the control feedback loop to mitigate the effects on the structure. The adaptive differentiator proposed in this paper is also different to the ones in $[9,21,22]$, since the latter papers consider a control design problem and seek to ensure the gains are as small as possible to avoid chattering. Although the work proposed in [21] considers an adaptive scheme which assumes an unknown bound on the disturbance (similar to the work proposed here), the adaptation rules and the Lyapunov analysis are quite different and the super-twisting gains continually adapt. The adaptation scheme proposed in this paper is motivated by the practical requirement for good reconstruction of the OFCs for a wide range of amplitudes/frequencies. For fault free and low amplitude/frequency OFCs, it is essential that the gains of the differentiator are as low as possible to provide good reconstruction in the noisy environment in which the actuator operates. However, during high amplitude/frequency OFCs, a larger gain is required to maintain sliding and to ensure a good reconstruction is still maintained.

\section{Adaptive Supertwist Differentiator}

Consider the scalar differential equation

$\dot{x}(t)=g(t, x, \xi)$

with measured output $y(t)=x(t)$. The function $\xi(t)$ represents potential faults which may occur within the system and is imprecisely known; however in fault free conditions $\xi \equiv 0$. Because of the presence of the faults the function $g(\cdot)$ is unknown but the time derivative of the function on the right hand side of (1) is assumed to be bounded by a scalar $\delta>0$. It is assumed that although $\xi(t)$ is unknown, some a-priori information is available from engineering understanding of the system such that a worst case (possibly very conservative) estimate $r_{\max }$ is available such that

$|\dot{g}(t, x, \xi)|<\delta<r_{\max }$

Consider a differentiator scheme of the form suggested in [14] with the following structure

$\dot{z}_{1}(t)=-\alpha(t)\left|e_{1}(t)\right|^{1 / 2} \operatorname{sign}\left(e_{1}(t)\right)+z_{2}(t)$
$\dot{z}_{2}(t)=-\beta(t) \operatorname{sign}\left(e_{1}(t)\right)$

where $e_{1}(t)=z_{1}(t)-x(t)$. Here $\alpha(t)$ and $\beta(t)$ are time varying scalars. Subtracting (1) from (3) yields

$\dot{e}_{1}(t)=-\alpha(t)\left|e_{1}(t)\right|^{1 / 2} \operatorname{sign}\left(e_{1}(t)\right)+z_{2}(t)-g(t, x)$

$\dot{z}_{2}(t)=-\beta(t) \operatorname{sign}\left(e_{1}(t)\right)$

Define a new variable $e_{2}(t)=z_{2}(t)-g(t, x, \xi)$, then (5)(6) can be written as

$\dot{e}_{1}(t)=-\alpha(t)\left|e_{1}(t)\right|^{1 / 2} \operatorname{sign}\left(e_{1}(t)\right)+e_{2}(t)$
$\dot{e}_{2}(t)=-\beta(t) \operatorname{sign}\left(e_{1}(t)\right)-\dot{g}(t, x, \xi)$

If a 2 nd order sliding motion is induced forcing $e_{1}(t)=$ $\dot{e}_{1}(t)=0$, from $(7), e_{2}(t)=0 \Rightarrow z_{2}(t)=g(t, x, \xi)$. Consequently $z_{2}(t)$ from (4) provides an estimate of $\dot{x}(t)$. In this paper, the gains $\alpha(t)$ and $\beta(t)$ are chosen as:

$\alpha(t)=\sqrt{2 L(t)}$

$\beta(t)=4 L(t)$

for some time varying scalar $L(t)$ which will be described in the sequel. Suppose the gain $L(t)$ has the form

$L(t)=r(t)+\ell>0$

where the variable $\ell$ is a fixed positive scalar while $r(t)$ (also positive) is adapted according to

$\dot{r}(t)= \begin{cases}\gamma\left|e_{1}(t)\right|^{1 / 2} & \text { if } r(t) \leq r_{\max } \\ 0 & \text { otherwise }\end{cases}$

where $\gamma>0$ is a positive design constant and the scalar $r_{\max }$ is the maximum value that $r(t)$ can take.

Whilst this scheme is intuitive and simple, it is not practical. In practical implementations, an ideal sliding is not achievable due to noise, and therefore the adaptation rule in (12) would result in $r(t)$ becoming unbounded. In the application which will be presented in the sequel, 
(12) has been modified to

$\dot{r}(t)= \begin{cases}\gamma D\left(\left|e_{1}(t)\right|^{1 / 2}\right) & \text { if } r(t) \leq r_{\max } \\ 0 & \text { otherwise }\end{cases}$

where the function $D(z): \mathbb{R} \mapsto \mathbb{R}$ is the dead-zone

$D(z)= \begin{cases}0 & \text { if }|z|<\epsilon \\ z & \text { otherwise }\end{cases}$

where $\epsilon$ is a positive scalar. The idea is to adapt the gains only when $\left|e_{1}(t)\right|^{1 / 2}$ unacceptably deviates from zero. The gain $r(t)$ will increase in magnitude according to (13) to force $e_{1}(t)$ back into a 'real sliding' regime.

Proposition 1 Under the assumption that the scalar $r_{\max }$ is chosen sufficiently large such that (2) holds for the class of faults represented by $\xi(t)$, using the adaptation rule (13) ensures the error system (7)-(8) and $r(t)$ remains bounded, and the variable $\left|e_{1}(t)\right|<a \epsilon$ in finite time, where $a$ is an arbitrary scalar satisfying $a>1$.

Proof: If at some time $t_{0}>0$ the adaptive gain $r(t)=$ $r_{\max }$, then $r(t)=r_{\max }$ for all $t>t_{0}$, and consequently the gains take the fixed values $\alpha(t)=\sqrt{2\left(\ell+r_{\max }\right)}$ and $\beta(t)=4\left(\ell+r_{\max }\right)$ for all $t>t_{0}$. Since $\ell+r_{\max }>$ $|\dot{g}(t, x, \xi)|$, the analysis in [16] shows $e_{1}(t)=e_{2}(t)=0$ for all $t>T>t_{0}$ for some (finite) time $T$ and the statement of the proposition is fulfilled. The remainder of the proof addresses the situation in which $r(t)<r_{\max }$ for all time.

Consider the following Lyapunov function candidate for the error system (7) and (8)

$$
\begin{array}{r}
V\left(\zeta, e_{r}\right)=\frac{2 \sqrt{2}}{\gamma} \frac{\left.\left(\bar{l}+e_{r}(t)\right)+\delta\right)}{\left(\bar{l}+e_{r}(t)\right)^{2}} e_{r}^{2}(t)+\frac{1}{\left(\bar{l}+e_{r}(t)\right)^{3 / 2}} \\
\times \zeta(t)^{\mathrm{T}}\left[\begin{array}{cc}
18\left(\bar{l}+e_{r}(t)\right) & -\sqrt{2\left(\bar{l}+e_{r}(t)\right)} \\
-\sqrt{2\left(\bar{l}+e_{r}(t)\right)} & 2
\end{array}\right] \zeta(t)(15)
\end{array}
$$

where $\bar{l}=\ell+\delta$, the error state

$e_{r}(t):=r(t)-\delta$

and the vector $\zeta^{\mathrm{T}}(t):=\left[\left|e_{1}(t)\right|^{1 / 2} \operatorname{sgn}\left(e_{1}(t)\right) e_{2}(t)\right]$. The motivation for the choice of Lyapunov function is that (15) can be equivalently written as

$$
\begin{aligned}
V\left(\zeta, e_{r}\right)= & \frac{1}{L^{3 / 2}(t)} \zeta(t)^{\mathrm{T}} P(t) \zeta(t) \\
& +\frac{\sqrt{2}}{2 \gamma} e_{r}(t)\left(4 L^{-1}(t)+\delta L^{-2}(t)\right) e_{r}(t)
\end{aligned}
$$

where

$$
P(t)=\frac{1}{2}\left[\begin{array}{cc}
4 \beta(t)+\alpha^{2}(t) & -\alpha(t) \\
-\alpha(t) & 2
\end{array}\right]
$$

and the scalars $\alpha(t), \beta(t)$ and $L(t)=\ell+\delta+e_{r}(t)$ are defined in (9)-(10). Because $0<r(0)+\ell \leq L(t) \leq$ $r_{\text {max }}+\ell$, it follows that $\beta(t)>0$ and $\operatorname{det}(P(t))>0$. A further consequence is the function $V\left(\zeta, e_{r}\right)$ from (15) is Lipschitz and positive definite. Taking the derivative of (17) yields

$$
\begin{aligned}
\dot{V}(t)= & \underbrace{\frac{d}{d t}\left(\frac{1}{L^{3 / 2}(t)} \zeta(t)^{\mathrm{T}} P(t) \zeta(t)\right)}_{\dot{V}_{1}} \\
& +\underbrace{\frac{d}{d t}\left(\frac{\sqrt{2}}{2 \gamma} e_{r}(t)\left(4 L^{-1}(t)+\delta L^{-2}(t)\right) e_{r}(t)\right)}_{\dot{V}_{2}}
\end{aligned}
$$

The first component can be expressed as

$$
\begin{aligned}
\dot{V}_{1}(t)= & \underbrace{\zeta^{\mathrm{T}}(t)\left(\frac{d}{d t}\left(\frac{1}{L^{3 / 2}(t)} P(t)\right)\right) \zeta(t)}_{\dot{V}_{1 a}} \\
& +\underbrace{\frac{1}{L^{3 / 2}(t)}\left(\dot{\zeta}(t)^{\mathrm{T}} P(t) \zeta(t)+\zeta(t)^{\mathrm{T}} P(t) \dot{\zeta}(t)\right)}_{\dot{V}_{1 b}}
\end{aligned}
$$

Consider initially the term $\dot{V}_{1 a}(t)$ in $(20)$ : substituting for $\alpha(t)$ and $\beta(t)$ from (9)-(10) gives

$$
\begin{aligned}
\dot{V}_{1 a}(t) & =\zeta^{\mathrm{T}}(t)\left(\frac{d}{d t} \frac{1}{2}\left[\begin{array}{cc}
18 L^{-1 / 2}(t) & -\sqrt{2} L^{-1}(t) \\
-\sqrt{2} L^{-1}(t) & 2 L^{-3 / 2}(t)
\end{array}\right]\right) \zeta(t) \\
& =\frac{1}{2} \zeta^{\mathrm{T}}(t) \underbrace{\left[\begin{array}{cc}
-9 L^{-3 / 2}(t) & \sqrt{2} L^{-2}(t) \\
\sqrt{2} L^{-2}(t) & -3 L^{-5 / 2}(t)
\end{array}\right]}_{\psi(t)} \dot{L}(t) \zeta(t)
\end{aligned}
$$

Notice that $\dot{L}(t) \geq 0$ since $\dot{L}(t)=\dot{r}(t)$, and from (12), $\dot{r}(t) \geq 0$ for all time. Since $L(t)>0$ it can be easily shown that $\psi(t)<0$ and consequently since $\dot{L}(t) \geq 0$, it follows

$\dot{V}_{1 a}(t)=\frac{1}{2} \zeta^{\mathrm{T}}(t) \psi(t) \dot{L}(t) \zeta(t) \leq 0$

Arguing as in [16], the term $\dot{V}_{1 b}(t)$ in $(20)$ is given by

$$
\begin{aligned}
\dot{V}_{1 b}(t)= & \frac{1}{L^{3 / 2}}\left(-\dot{g}(t)\left(-\alpha(t)\left|e_{1}\right|^{1 / 2} \operatorname{sgn}\left(e_{1}\right)+2 e_{2}\right)\right. \\
& \left.-\frac{1}{\left|e_{1}\right|^{1 / 2}} \zeta^{\mathrm{T}} Q \zeta\right)
\end{aligned}
$$

where

$Q(t)=\frac{\alpha(t)}{2}\left[\begin{array}{cc}2 \beta(t)+\alpha^{2}(t) & -\alpha(t) \\ -\alpha(t) & 1\end{array}\right]$ 
Since it is assumed that $\dot{g}(t, x)$ is bounded by $\delta$

$$
\begin{aligned}
& \dot{V}_{1 b}(t) \leq-\frac{\alpha}{2 L^{3 / 2}\left|e_{1}\right|^{1 / 2}}\left(\left(2 \beta+\alpha^{2}-2 \delta\right)\left|e_{1}\right|+e_{2}^{2}\right. \\
& \left.-2 \alpha\left|e_{1}\right|^{1 / 2} \operatorname{sgn}\left(e_{1}\right) e_{2}-\frac{4}{\alpha} \operatorname{sgn}\left(e_{1}\right) \dot{g}\left|e_{1}\right|^{1 / 2} \operatorname{sgn}\left(e_{1}\right) e_{2}\right)
\end{aligned}
$$

If

$$
\tilde{e}_{2}:=e_{2}-\left(\alpha+\frac{2}{\alpha} \operatorname{sgn}\left(e_{1}\right) \dot{g}\right)\left|e_{1}\right|^{1 / 2} \operatorname{sgn}\left(e_{1}\right)
$$

then replacing $e_{2}$ with $\tilde{e}_{2}$ in (25) means

$$
\begin{aligned}
\dot{V}_{1 b}(t) \leq & -\frac{\alpha}{2 L^{3 / 2}\left|e_{1}\right|^{1 / 2}}\left(\left(2 \beta+\alpha^{2}-2 \delta\right.\right. \\
& \left.\left.-\left(\alpha+\frac{2}{\alpha} \operatorname{sgn}\left(e_{1}\right) \dot{g}\right)^{2}\right)\left|e_{1}\right|+\tilde{e}_{2}^{2}\right)
\end{aligned}
$$

Finally it follows that

$$
\begin{aligned}
\dot{V}_{1 b}(t) \leq & -\frac{\alpha}{2 L^{3 / 2}\left|e_{1}\right|^{1 / 2}}\left(\left(2 \beta+\alpha^{2}-2 \delta\right.\right. \\
& \left.\left.-\left(\alpha+\frac{2}{\alpha} \delta\right)^{2}\right)\left|e_{1}\right|+\tilde{e}_{2}^{2}\right)
\end{aligned}
$$

since for any value of $\dot{g}$ satisfying $|\dot{g}| \leq \delta$,

$$
\left(\alpha+\frac{2}{\alpha} \operatorname{sgn}\left(e_{1}\right) \dot{g}\right)^{2} \leq\left(\alpha+\frac{2}{\alpha} \delta\right)^{2}
$$

because $\alpha>0$. Substituting for $\alpha$ and $\beta$ from (9) and (10) in (27) yields

$$
\begin{aligned}
\dot{V}_{1 b}(t) & \leq-\frac{\alpha}{2 L^{3 / 2}\left|e_{1}\right|^{1 / 2}}\left(\tilde{e}^{2}+\left(8 L-6 \delta-2 \delta^{2} L^{-1}\right)\left|e_{1}\right|\right) \\
& \equiv-\frac{\alpha}{2 L^{3 / 2}\left|e_{1}\right|^{1 / 2}}\left(\tilde{e}^{2}+\frac{2}{L}(4 L+\delta)(L-\delta)\left|e_{1}\right|\right)(28)
\end{aligned}
$$

Using (9), (16) and the fact that $L-\delta=(r+\ell-\delta)=$ $\ell+e_{r},(28)$ can be written as

$$
\begin{aligned}
\dot{V}_{1 b}(t) \leq & -\frac{\alpha(t)}{2 L^{3 / 2}(t)\left|e_{1}\right|^{1 / 2}} \tilde{e}^{2} \\
& -\sqrt{2}\left(4 L^{-1}+\delta L^{-2}\right)\left(\ell+e_{r}\right)\left|e_{1}\right|^{1 / 2}
\end{aligned}
$$

Now consider $\dot{V}_{2}$ from (19): simple calculations give

$$
\begin{aligned}
\dot{V}_{2}(t)= & \frac{\sqrt{2}}{\gamma}\left(4 L^{-1}+\delta L^{-2}\right) e_{r} \gamma D\left(\left|e_{1}\right|^{1 / 2}\right) \\
& -\frac{1}{\sqrt{2} \gamma}\left(e_{r}\left(4 L^{-2}+2 \delta L^{-3}\right) \dot{L} e_{r}\right)
\end{aligned}
$$

From (22), (29) and (30), the Lyapunov derivative is

$$
\dot{V}(t)=\dot{V}_{1 a}(t)+\dot{V}_{1 b}(t)+\dot{V}_{2}(t)
$$

$$
\begin{aligned}
\leq & \frac{1}{2} \zeta^{\mathrm{T}} \psi(t) \dot{L} \zeta-\frac{\alpha}{2 L^{3 / 2}\left|e_{1}\right|^{1 / 2}} \tilde{e}^{2} \\
& -\sqrt{2}\left(4 L^{-1}+\delta L^{-2}\right)\left(\ell+e_{r}\right)\left|e_{1}\right|^{1 / 2} \\
& +\sqrt{2}\left(4 L^{-1}+\delta L^{-2}\right) e_{r} D\left(\left|e_{1}\right|^{1 / 2}\right) \\
& -\frac{1}{\sqrt{2} \gamma}\left(4 L^{-2}+2 \delta L^{-3}\right) \dot{L} e_{r}^{2}
\end{aligned}
$$

If $\ell+e_{r}>0$ (or equivalently if $r(t)>\delta-\ell>0$ ) then $\dot{V} \leq 0$. Otherwise there are two cases to consider:

a) If $\left|e_{1}\right|^{1 / 2}>\epsilon$, then $D\left(\left|e_{1}\right|^{1 / 2}\right)=\left|e_{1}\right|^{1 / 2}$ and so substituting in (31) gives

$$
\begin{aligned}
\dot{V}(t) \leq & -\frac{\alpha}{2 L^{3 / 2}\left|e_{1}\right|^{1 / 2}} \tilde{e}^{2}-\sqrt{2}\left(4 L^{-1}+\delta L^{-2}\right) \ell\left|e_{1}\right|^{1 / 2} \\
& -\frac{1}{\sqrt{2} \gamma}\left(4 L^{-2}+2 \delta L^{-3}\right) \dot{L} e_{r}^{2}
\end{aligned}
$$

Consequently $\dot{V}(t) \leq 0$ since $\psi(t)<0$ and $\dot{L} \geq 0$.

b) If $\left|e_{1}\right|^{1 / 2}<\epsilon, D\left(\left|e_{1}\right|^{1 / 2}\right)=0$ and substituting in (31)

$$
\begin{aligned}
\dot{V}(t) \leq & -\frac{1}{\sqrt{2} L\left|e_{1}\right|^{1 / 2}} \tilde{e}^{2}-\frac{1}{\sqrt{2} \gamma}\left(4 L^{-2}+2 \delta L^{-3}\right) \dot{L} e_{r}^{2} \\
& -\sqrt{2}\left(4 L^{-1}+\delta L^{-2}\right)\left|e_{1}\right|^{1 / 2}\left(\ell+e_{r}\right)
\end{aligned}
$$

after substituting for $\alpha(t)$ from (9). Recall $\dot{e}_{r}(t)=\dot{r}(t) \geq$ 0 and so $e_{r}(t) \geq e_{r}(0)$ for all $t \geq 0$. Under the assumption that $r(0)<\delta$ it follows

$$
-e_{r}(t) \leq-e(0)=\delta-r(0)>0
$$

Also since $L(t)=r(t)+\ell$ and $\dot{r} \geq 0$ it follows $L(t) \geq L(0)$ for all $t \geq 0$ and consequently

$$
\begin{aligned}
-\sqrt{2}\left(4 L(t)^{-1}\right. & \left.+\delta L(t)^{-2}\right)\left|e_{1}(t)\right|^{1 / 2} e_{r}(t) \\
& \leq \sqrt{2} \epsilon\left(4 L(0)^{-1}+\delta L(0)^{-2}\right)(\delta-r(0))
\end{aligned}
$$

Therefore for $\left|e_{1}\right|^{1 / 2}<\epsilon$, from (33), it follows that

$$
\begin{aligned}
\dot{V}(t) \leq & \sqrt{2}\left(4 L(0)^{-1}+\delta L(0)^{-2}\right) \epsilon(\delta-r(0)) \\
& -\frac{1}{\sqrt{2}\left(r_{\max }+\ell\right) \epsilon} \tilde{e}^{2}
\end{aligned}
$$

Define $\epsilon_{0}=\left(2 \epsilon^{2}\left(r_{\max }+\ell\right)\left(4(r(0)+\ell)^{-1}+\delta(r(0)+\right.\right.$ $\left.\left.\ell)^{-2}\right)(\delta-r(0))\right)^{1 / 2}$ then it follows if $\tilde{e}^{2}>\epsilon^{2}$ then $\dot{V} \leq 0$ in $(34)$.

From the preceding argument, outside

$\mathcal{R}=\left\{\left.\left(e_{1}, \tilde{e}, e_{r}\right)|| e_{1}\right|^{1 / 2}<\epsilon,|\tilde{e}|<\epsilon_{0},-\delta \leq e_{r} \leq-\ell\right\}($

the derivative $\dot{V} \leq 0$ and consequently the states $\left(e_{1}, \tilde{e}, e_{r}\right)$ remain bounded. Define another hyper rectangle $\mathcal{R}_{a}=\left\{\left.\left(e_{1}, \tilde{e}, e_{r}\right)|| e_{1}\right|^{1 / 2}<a \epsilon,|\tilde{e}|<a \epsilon_{0}, \quad-a \delta \leq\right.$ 
$\left.e_{r} \leq-\ell / a\right\}$ where the scalar $a>1$. Then by construction $\mathcal{R} \subset \mathcal{R}_{a}$ and outside the set $\mathcal{R}_{a}$, the Lyapunov derivative $\dot{V}(t) \leq-\epsilon_{a}$ where $\epsilon_{a}>0$ is a positive constant depending on $a$ (with the property that $\epsilon_{a}$ tends to zero from above as $a \rightarrow 1$ from above). Consequently $\mathcal{R}_{a}$ is entered in finite time and hence $\left|e_{1}(t)\right|<a \epsilon$ in finite time.

Remark: In the next section, the reasoning behind the use of an adaptive scheme, even though an upper bound on $\dot{g}(t, x, \xi)$ is known, will be given in terms of a real engineering OFC application. In the situation when $\xi(t)$ a small incipient low frequency fault, small values for $\alpha(t)$ and $\beta(t)$ are preferable, because the effect of noise on the measurement $y(t)$ and its subsequent effect on the estimate $\dot{y}(t)$ (which is used in the FDI scheme to estimate $\xi(t))$, will be mitigated. The transfer of noise via unnecessarily large values for the gains $\alpha(t)$ and $\beta(t)$ will tend to mask small incipient low frequency faults, making them harder to detect. However in order to preserve a sliding motion, and hence the accuracy of the estimate of $\dot{y}(t)$ in the presence of high amplitude high frequency faults, large values of $\alpha(t)$ and $\beta(t)$ must be employed. Consequently in order to preserve the fidelity of the estimate of $\dot{y}(t)$ for a wide range of high and low frequency faults, the gains must be allowed to adapt.

Remark: In a situation when no adaptation takes place and the gains remain fixed, the observer behaves exactly as a Levant differentiator [14] and so all the optimal properties of that scheme with respect to noise and sampling are preserved.

\section{OFC Estimation - ADDSAFE Benchmark}

The Advanced Fault Diagnosis for Sustainable Flight Guidance and Control (ADDSAFE) project is an European funded collaboration which aims to demonstrate the applicability of advanced fault detection and diagnosis (FDD) methods for aircraft to support the development of sustainable aircraft. The main objectives are to improve FDD methods to support new 'green' technologies allowing optimization of the aircraft structural design, improving aircraft performance and reducing the environmental footprint [15]. The ADDSAFE benchmark model provided by AIRBUS [10] represents a high fidelity generic twin engine civil commercial transport aircraft. It comprises a rigid-body aircraft model with a complex aerodynamic database, a full set of control surfaces, highly detailed nonlinear actuators and sensor models, and realistic levels of sensor noise.

One of the fault detection problems to be investigated in ADDSAFE is the Oscillatory Failure Case (OFC). An OFC is a type of EFCS failure which can cause unacceptably high vibration and significant increases in the structural load due to erroneous oscillation [11]. The idea is to estimate actuator rod speed (using the adaptive sliding mode super-twisting differentiator) which is needed for estimating OFC (through the manipulation of the analytical mathematical model of the actuator).

\subsection{Modelling of hydraulic actuator}

The hydraulic actuator model which will be considered here is from $[10,11]$ and is given by

$\dot{x}(t)=V_{c}(t)\left(\frac{\Delta_{p}(t)-\operatorname{sign}(i(t)) \frac{F_{a e r o}(t)}{S}}{\Delta_{p_{\text {ref }}}+\frac{K_{d}(t)}{S} V_{c}^{2}(t)}\right)^{\frac{1}{2}}$

where nominally

$V_{c}(t)=K_{c} i(t)$

and $K_{c}$ is a conversion factor from electrical current $(\mathrm{mA})$ to speed $(\mathrm{mm} / \mathrm{s})$. The current $i(t)$ is given by

$i(t)=K(u(t)-x(t))$

where $K$ is the (fixed) servo control gain. This model is embedded in the benchmark model. The signal $u(t)$ is the commanded rod position from the FCC and $x(t)$ is the hydraulic actuator rod position. The fixed constants are $S$ which is the piston surface area and $\Delta_{p_{\text {ref }}}$ which is the differential pressure corresponding to the maximum rod speed. The parameters which depend on varying operational conditions (e.g. fluid temperature and/or the number of actuators used simultaneously on a given hydraulic circuit) are $F_{\text {aero }}(t)$ which represent the aerodynamic forces applied on the control surface, $\Delta_{p}(t)$ which is the actual hydraulic pressure delivered to the actuator and $K_{d}(t)$ which is the adjacent actuator damping coefficient.

\subsection{OFC modelling}

An OFC is caused by a faulty digital component in the Electrical Flight Control System EFCS (aka fly by wire), which generates unwanted sinusoidal signals in the actuator loop between the Flight Control Computer (FCC) and the control surface [11] (see Figure 1). The oscillations propagate within the loop generating extra structural loads. When coupled with the aeroelastic behaviour of the aircraft they can create excessive loads and vibrations (and could generate resonances in the natural modes of the structure) [11]. Similar to [11], only an OFC located in the servo control loop is considered in this paper. Specifically, it is assumed that the OFC originates from the analogic output signal between the FCC and the actuator (See Figure 1). In the ADDSAFE model, the OFC affects the computed/desired rod speed $V_{c}(t)$ so that

$V_{c}(t)= \begin{cases}V_{0}(t) & \text { nominal } \\ V_{0}(t)+K_{c} f_{l i q}(t) & \text { liquid OFC } \\ K_{c} f_{\text {sol }}(t) \text { i.e. } V_{0}(t)=0 & \text { solid OFC }\end{cases}$ 


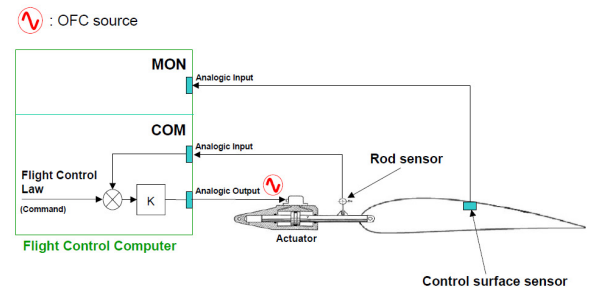

Fig. 1. Source of OFC in the ADDSAFE benchmark problem

where $V_{0}(t)=K_{c} K(u(t)-x(t))$. The OFC signals are considered as sinusoids with amplitude and frequency uniformly distributed over the range $1-10 \mathrm{~Hz}$ [11]. Due to the low pass characteristics of the actuator, an OFC with frequency beyond $10 \mathrm{~Hz}$ has no effect on control surface oscillation. As shown in (39) the liquid OFC behaves as an additive fault, and the OFC signal adds to the desired position from the FCC, and hence the control surface tracks the corrupted demand signal. In the case of a solid OFC, the demanded surface position is totally replaced by the OFC signal and the control surface does not respond to the desired rod position command - i.e., it is totally 'disconnected' from the FCC, and instead performs a pure periodic motion. Consequently any attempt to damp the oscillation does not have any impact.

\subsection{OFC Estimation}

To obtain an estimate of the OFC, the nonlinear equations (36), (38) and (39) have been rearranged to obtain an expression of the OFC signal. Consider initially the liquid $O F C$ case. By algebraic rearrangement, (36) and (39) are equivalent to

$$
f_{l i q}(t)=\frac{\dot{x}(t)\left(\frac{\Delta_{p_{\text {ref }}}}{\Delta_{p}(t)-\operatorname{sign}(i(t)) \frac{F_{a e r o}(t)}{S}-\dot{x}^{2}(t) \frac{K_{d}(t)}{S}}\right)^{\frac{1}{2}}-V_{0}(t)}{K_{c}}
$$

All the variables on the right hand side of (40) are available (measured) or can be estimated except for the actuator rod speed $\dot{x}(t)$. For the solid OFC case, since $V_{0}=0$ in (39), similar arguments give

$f_{\text {sol }}(t)=\frac{\dot{x}(t)\left(\frac{\Delta_{p_{\text {ref }}}}{\Delta_{p}(t)-\operatorname{sign}(i(t)) \frac{F_{a e r o}(t)}{S}-\dot{x}^{2}(t) \frac{K_{d}(t)}{S}}\right)^{\frac{1}{2}}}{K_{c}}$

The idea in this paper is to use the adaptive supertwisting differentiator from Section 2 to provide a robust estimate of rod speed from the measurement of rod position $x(t)$. Once $\dot{x}(t)$ is estimated, the OFC can be estimated using (40) and (41) since all the values on the right hand side are known.

In fault free conditions, 'small gains' are sufficient to ensure a good estimate of rod speed while ensuring that the effect of noise is not accentuated. However, when an
OFC occurs, for small values of $\alpha$ and $\beta$ sliding might be broken thus compromising the estimate of the rod speed and OFC estimation. In this case, larger gains are required to ensure sliding is maintained to provide a good rod speed and OFC estimate.

\section{Simulations}

The proposed scheme has been tested on a high fidelity aircraft model provided by AIRBUS [10]. The high fidelity nonlinear actuator model is parameterized by the varying parameters $\left(\Delta_{p}, F_{\text {aero }}\right.$ and $\left.K_{d}\right)$ which change based on the operating conditions. For observer design purposes, these parameters are assumed to be fixed at their mean values. The simulations have been conducted at an altitude of $20000 \mathrm{ft}$, a speed of $220 \mathrm{kts}$, a weight of 230 tonnes and centre of gravity of $37 \%$ MAC. For the simulations in this paper, the control surface considered is the left elevator and the OFC is considered to originate from the analogic output of the FCC. The OFC at this source will corrupt the electrical signal which provides the desired actuator position to the solenoid valve of the actuator. The challenge specified from ADDSAFE requires an $\mathrm{OFC}$ to be detected within 3 periods.

The sliding mode super-twisting differentiator design parameters from (11) and (13) used in the simulation are $\gamma=3 \times 10^{4}, \ell=50$ and $\epsilon=0.5$. In the following simulations $r_{\max }=5000 \mathrm{~mm} / \mathrm{s}^{2}$ has been chosen. This value corresponds to the maximum rate of acceleration of the actuator rod because $\dot{g}(x, u, \xi)=\ddot{x}(t)$. This choice is very conservative and well beyond what can be physically attained by the actuator according to the manufacturer's data sheet. It is also well beyond the value obtained from simple worst case calculations associated with the most aggressive fault (a sinusoidal input of amplitude 10 at a frequency of $10 \mathrm{~Hz}$ ) occurring instantaneously with a severe step-input pilot command. As will be seen in the later simulations, this value of $r_{\max }$ is an order of magnitude larger that the actual values $r(t)$ ever takes during the simulation tests. To ensure a design which satisfies the restrictions on the computational power load associated with the flight computer, (running at $100 \mathrm{hz}$ ), the simulations were performed using a solver with a fixed time step of $0.01 \mathrm{~s}$. For added realism, sensor and process noise have been included in the simulation.

\subsection{Simulation Results}

For consistency and for comparison, the OFC is set to occur at 20.3sec and the excitation which has been considered for the ADDSAFE benchmark problem is the ' $\alpha$ protection' manoeuvre (see Figure 2(a)). This aggressive manoeuvre is characterized by a sudden large pilot stick movement at 20sec, which triggers the angle of attack protection system (currently a built-in feature of the controller) to prevent an excessively high angle of attack 
(see Figure 2(b)). For brevity, although a wide range of OFC amplitudes and frequencies have been tested, the results shown here represent the extreme cases of low and high amplitude/frequency to highlight the performance of the proposed scheme. The low amplitude OFC case shows the smallest amplitude the scheme can detect (especially when masked by the noise in the system) while the high frequency OFC case represents a challenge to detect the failure within the required time.

Figure 2 shows the fault free scenario. Figure 2(a) shows the sudden large input applied to the pilot longitudinal stick to excite the system. The effect of the stick input on the left elevator deflection is also given in Figure 2(a). In nominal fault free conditions, the actual control surface deflection (blue solid line), is overlapped by the demand signal from the FCC (red dash line). The change in the operating condition is shown in Figure 2(b). Figure 2(c) shows the performance of the sliding mode supertwisting differentiator in the nominal case with the error signal $e_{1}$ close to zero and no adaptation taking place $(L(t)$ remains constant). Figure 2(d) shows a good estimate of rod speed $\dot{x}(t)$ (solid blue line) compared to the actual rod speed (red dashed line). Figure 2(d) also shows no OFC is present. This is confirmed by the detection signals (boolean) shown in Figure 2(e).

Figures 3-4 show the results for a liquid OFC type failure for low amplitude/frequency and high amplitude/frequency OFC cases. Figure 3 shows an OFC with an amplitude of $0.5 \mathrm{deg}$ at a frequency of $0.5 \mathrm{~Hz}$. Figure 3(a) shows the effect of a liquid OFC on the left elevator deflection (blue solid line) after 20.3sec whereby the OFC signal adds to the demanded signal (red dashed line) from the FCC. Figure 3(c) shows that for this combination of low amplitude and frequency, no adaptation to the super-twisting gain $L(t)$ is required as no degradation in the sliding motion occurs $\left(e_{1}(t)\right.$ close to zero). Figure 3(d) shows a good estimate of rod speed $\dot{x}$ (solid blue line) as it overlaps the actual rod speed (red dashed line). Figure 3(d) also shows a good estimate of the OFC (blue solid line) compared with the actual OFC (red dashed line). Note that the green line at \pm 1 on the plot of the OFC estimation represents the threshold which has been used in the detection logic (essentially oscillation counting similar to the one in [11]). The detection logic confirms the presence of an OFC after 2.5 cycles (Figure 3(b)).

Figure 4 shows the results for a liquid OFC with amplitude $1 \mathrm{deg}$ and a frequency of $7 \mathrm{~Hz}$. Zoomed-in plots from 19-23sec are presented due to the high frequency associated with the OFC. Figure 4(c) shows that when the OFC occurs at $20.3 \mathrm{sec}$, the quality of sliding degrades and the gain $L(t)$ increases to re-establish sliding. Figure 4(d) shows the estimate of both the rod speed and the OFC. During the period of sliding degradation, the rod speed and the OFC estimate slightly degrade, but once the gain $L(t)$ is sufficiently big, the quality of the estimation quickly recovers and both the rod speed and OFC
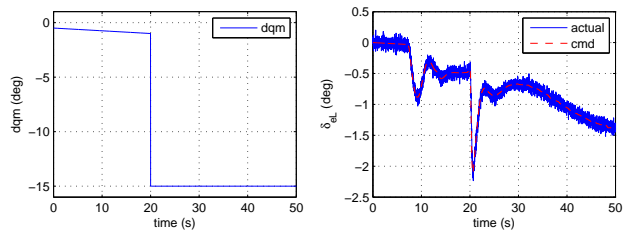

(a) pilot stick input and control surface deflection
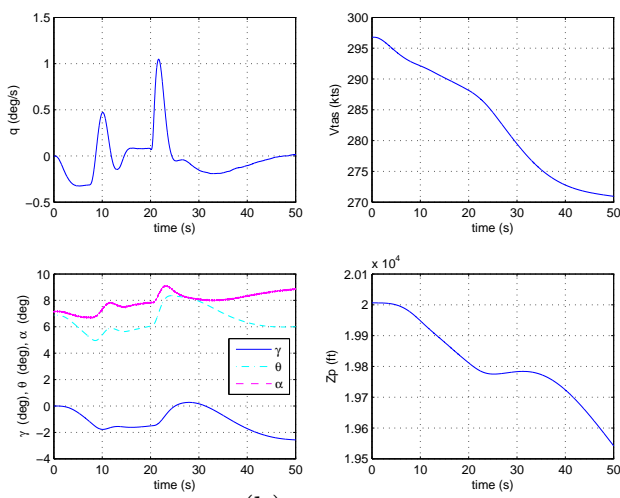

(b) states
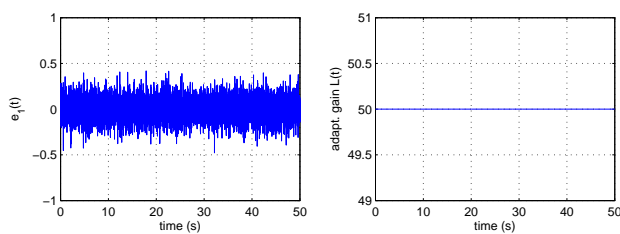

(c) supertwist performance
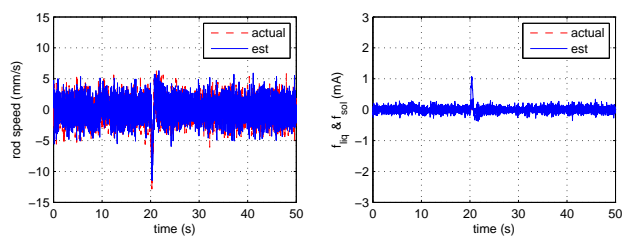

(d) OFC estimations
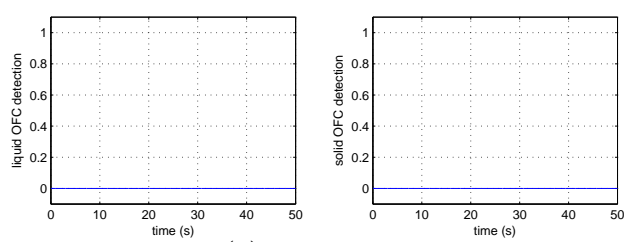

(e) detections

Fig. 2. Fault free condition

estimate (blue solid line) overlap the actual rod speed and OFC (red dashed line). The green line at \pm 400 in the plot of $L(t)$ (Figure 4(c)) is a threshold which has been used in conjunction with the oscillation counting for $\mathrm{OFC}$ detection. The detection logic confirms the presence of an OFC either by oscillation counting or when the threshold of $L(t)$ has been exceeded. In the event of a high frequency OFC, the gain $L(t)$ increases. As shown in Figure 4(b), the detection of the OFC is immediate, and much less than the 3 cycle detection requirement. 


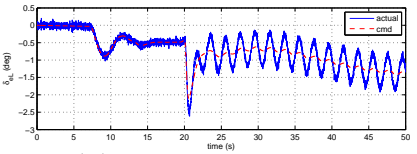

(a) control surface
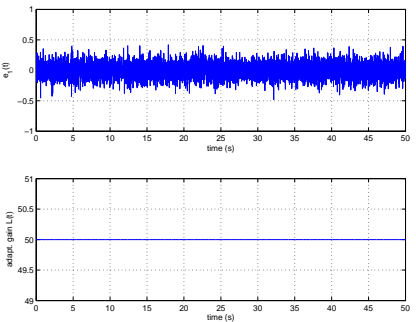

(c) supertwist performance

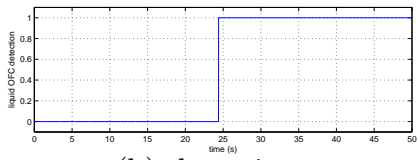

(b) detections
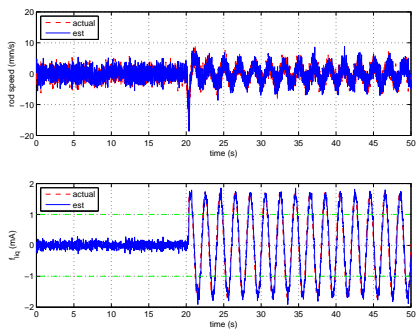

(d) estimations
Fig. 3. Liquid OFC (amplitude 0.5, frequency 0.5) (a) control surface
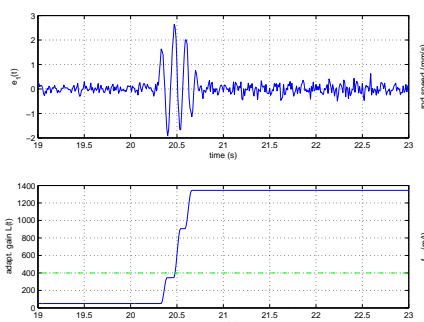

(c) supertwist performance

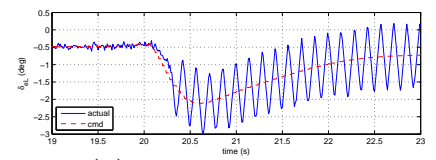

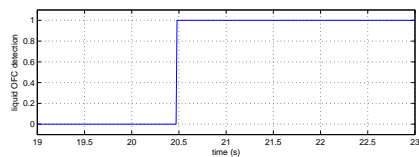

(b) detections
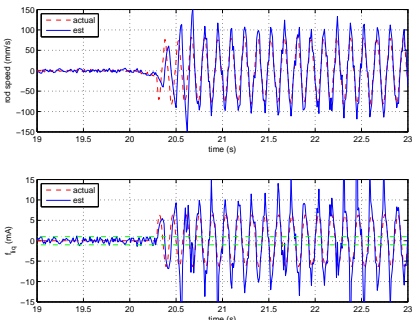

(d) estimations
Fig. 4. Liquid OFC (amplitude 1.0, frequency 7.0)

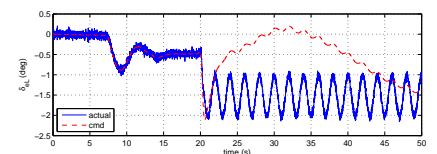

(a) control surface
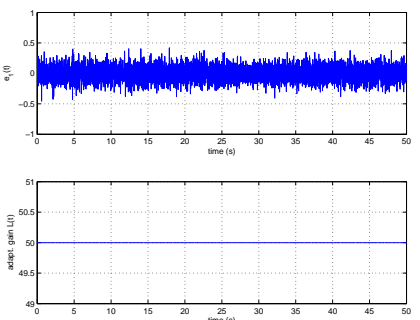

(c) supertwist performance

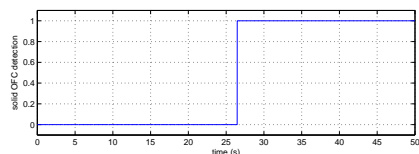

(b) detections
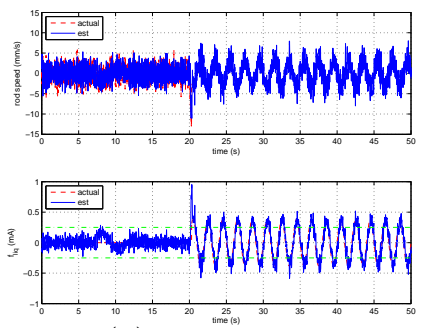

(d) estimations
Fig. 5. Solid OFC (amplitude 0.5, frequency 0.5)

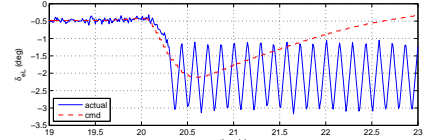

(a) control surface
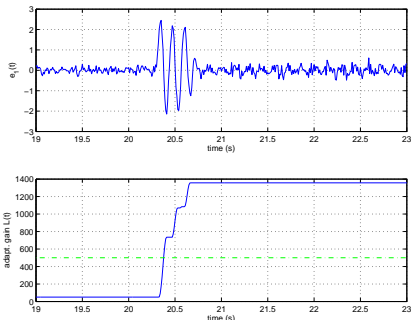

(c) supertwist performance

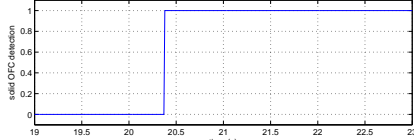

(b) detections
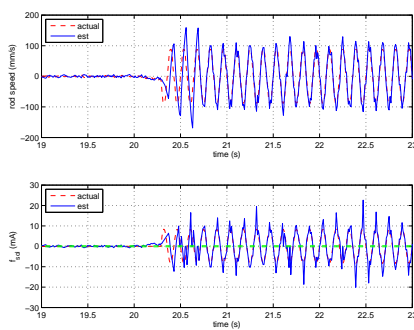

(d) estimations
Fig. 6. Solid OFC (amplitude 1.0, frequency 7.0)

Figures 5-6 show solid OFC type failures for two different amplitudes and frequencies. Figure 5 shows a low frequency/amplitude OFC case (amplitude 0.5deg at a frequency of $0.5 \mathrm{~Hz}$ ) while Figure 6 shows a high frequency/amplitude OFC case (amplitude 1deg at a frequency of $7 \mathrm{~Hz}$ ). Figure $5(\mathrm{a})$ shows the effect of the low amplitude/frequency OFC on the left elevator. It can be seen that after $20.3 \mathrm{sec}$ (immediately after the $\alpha$ protection manoeuvre), the commanded signal (red dashed line) is totally replaced by the OFC signal (blue solid line). After this point, the elevator does not respond to any commanded signal from the FCC. Figure 5(c) shows that the error signal $e_{1}$ remains close to zero and there is no super-twisting gain adaptation required for this level of OFC. Figure 5(d) shows a good estimate of the rod speed is obtained as the estimate (blue solid line) overlaps the actual (red dashed line) rod speed. Figure 5(d) also shows a good OFC estimate (blue solid line) from equation (41) as a result of the good rod speed estimate. The green line at \pm 0.25 on the plot of the OFC estimate is a threshold used for oscillation counting to confirm an OFC has occurred (Figure 5(b)).

Figure 6 shows a solid OFC of amplitude 1deg and frequency of $7 \mathrm{~Hz}$. Zoomed-in plots (19-23sec) are shown to illustrate the effectiveness of the scheme. Figure 6(a) shows the effect of a solid OFC on the left elevator. Again the OFC totally replaces the command signal from the FCC (red dashed line) when the OFC occurs at 20.3sec. Figure 6(c) shows the gain $L(t)$ adapting to ensure that sliding is reestablished (see $e_{1}(t)$ signal) after the OFC occurs and to ensure good estimates (blue solid lines) of both rod speed and the OFC as shown in Figure 6(d). These good estimates are obtained in the presence of noise. Figure 6(b) shows an almost immediate detection which confirms the presence of the OFC after 20.3sec. Here, (apart from the typical oscillation counting) the adaptive gain $L(t)$ was used as part of the detection logic. The idea is to use the higher gains required to reestablish sliding, as a measure of severity of the high frequency OFC. As shown in Figure 6(c), the green line is 
the threshold set for the high frequency OFC detection, which for a solid OFC is set at 500. In Figure 4(c) it can be seen that in the formulation adopted here the gain $L(t)$ can only increase. In some situations this would be a disadvantage: for example in a situation where the super-twist structure is used for feedback control, it is extremely useful to keep the gains as small as possible to help prevent chattering. Here this is not a significant issue because the observer is used purely for monitoring purposes and is not part of a control feedback loop.

\section{Conclusions}

This paper has presented a new adaptive super-twisting differentiator scheme in which the stability analysis is based on a Lyapunov approach. This adaptive differentiator has been used as a basis for a fault reconstruction scheme. The super-twisting gains are allowed to adapt to maintain sliding in a situation in which a severe fault is present in the system, but remain low in the fault free case. This is motivated by the need for good estimation even in the presence of noise, requiring the supertwisting gains to be as low as possible. When a severe fault occurs in the system, sliding is degraded and the super-twisting gains are allowed to increase to regain a sliding motion. The efficacy of the proposed method is shown by applying it to an OFC detection problem. Simulations based on the full nonlinear model of the aircraft using a highly detailed model of the actuators have been carried out for both liquid and solid OFC cases. The results show good estimates of the OFCs allowing their detection within the required specified time.

\section{References}

[1] E. Alcorta-Garcia, A. Zolghadri, and P. Goupil. A novel non-linear observer-based approach to oscillatory failure detection. In European Control Conference, pages 1901-1906, 2009 .

[2] E. Alcorta-Garcia, A. Zolghadri, P. Goupil, L. Lavigne, and P. Simon. Nonlinear observer-based OFC detection for A380 aircraft. In Proceedings of the IFAC Symposium SAFEPROCESS '09, Barcelona, pages 47-52, 2009.

[3] H.M. Besch, H. G. Giesseler, and J. Schuller. Impact of electronic flight control system (EFCS) failure cases on structural design loads. Agard report 815, loads and requirements for military aircraft, 1996.

[4] W. Chen and M. Saif. Actuator fault diagnosis for uncertain linear systems using a high-order sliding-mode robust differentiator (HOSMRD). International Journal of Robust and Nonlinear Control, 18:413-426, 2008.

[5] C. Edwards, S. Spurgeon, and R. Patton. Sliding mode observers for fault detection. Automatica, 36:541-553, 2000.

[6] T. Floquet, C. Edwards, and S. K. Spurgeon. On sliding mode observers for systems with unknown inputs. International Journal of Adaptive Control and Signal Processing, 21:638656, 2007.
[7] L. Fridman, J. Davila, and A. Levant. High-order sliding mode observation of linear systems with unknown inputs. In Proceedings of the IFAC World Congress, Seoul, pages 47794790, 2008.

[8] L. Fridman, Y. Shtessel, C. Edwards, and X.G. Yan. Higherorder sliding-mode observer for state estimation and input reconstruction in nonlinear systems. International Journal of Robust and Nonlinear Control, 18:399412, 2008.

[9] T. Gonzalez, J Moreno and L. Fridman. Variable gain super-twisting sliding mode control. IEEE Transactions on Automatic Control, 57, 2012.

[10] P. Goupil. AIRBUS benchmark description. Technical Report D1.1.3, AIRBUS OPERATIONS SAS, 2010.

[11] P. Goupil. Oscillatory failure case detection in the A380 electrical flight control system by analytical redundancy. Control Engineering Practice, 18(9):1110-1119, 2010.

[12] H.K. Khalil. Nonlinear Systems. Prentice Hall, Englewood Cliffs NJ., 1992.

[13] L. Lavigne, A. Zolghadri, P. Goupil, and P. Simon. Oscillatory failure case detection for new generation AIRBUS aircraft: a model-based challenge. In Proceedings of the IEEE CDC Cancun, Mexico, pages 1249-1254, 2008.

[14] A. Levant. Robust exact differentiation via sliding mode technique. Automatica, 34(3):379-84, 1998.

[15] A. Marcos. Advanced fault diagnosis for sustainable flight guidance and control. In 6th European Aeronautics Days, AERODAYS, Madrid, Spain, 2011.

[16] J. A. Moreno and M. Osorio. Strict Lyapunov functions for the super-twisting algorithm. IEEE Transactions on Automatic Control, 57, pages 1035-1040, 2012.

[17] R. Pons, C. Jauberthie, L. Trav-Massuys, and P. Goupil. Control surfaces oscillatory failures identification using interval analysis. In The 19th International Workshop on Principles of Diagnosis (DX-08), 2008.

[18] H. Sachs. Fault Investigation and Robust Failure Detection of Oscillating Aircraft Actuation Systems Using Analytical Redundancy. Shaker Verlag GmbH, 2010.

[19] M. Saif, W. Chen, and Q. Wu. High order sliding mode observers and differentiators-application to fault diagnosis problem. In G. Bartolini, L. Fridman, A. Pisano, and E. Usai, editors, Modern Sliding Mode Control Theory, volume 375 of LNCIS, pages 321-344. Springer-Verlag, 2008.

[20] Y. Shtessel, C. Hall, S. Baev, and J. Orr. Flexible modes control using sliding mode observers: Application to Ares I. In Proceedings of the Conference on Guidance, Navigation and Control, Toronto, Canada, 2010.

[21] Y. Shtessel, F. Plestan, and M. Taleb. Lyapunov design of adaptive super-twisting controller applied to a pneumatic actuator. In Proceedings of 18th IFAC World Congress Milano (Italy), 2011.

[22] Y. B. Shtessel, J. A. Moreno, F. Plestan, L. M. Fridman, and A. S. Poznyak. Super-twisting Adaptive Sliding Mode Control: A Lyapunov Design. In Proceedings of the 49th IEEE CDC, Atlanta, pages 5109-5113, 2010. 